\title{
The immune response against flaviviruses
}

\author{
Jose Luis Slon Campos ${ }^{1}$, Juthathip Mongkolsapaya ${ }^{1,2}$ and Gavin R. Screaton ${ }^{3 \star}$
}

\begin{abstract}
Arthropod-borne flaviviruses are important human pathogens that cause a diverse range of clinical conditions, including severe hemorrhagic syndromes, neurological complications and congenital malformations. Consequently, there is an urgent need to develop safe and effective vaccines, a process requiring better understanding of the immunological mechanisms involved during infection. Decades of research suggest a paradoxical role of the immune response against flaviviruses: although the immune response is crucial for the control, clearance and prevention of infection, poor clinical outcomes are commonly associated with virus-specific immunity and immunopathogenesis. This relationship is further complicated by the high homology among viruses and the implication of cross-reactive immune responses in protection and pathogenesis. This Review examines the dual role of the adaptive immune response against flaviviruses, particularly emphasizing the most recent findings regarding cross-reactive $T$ cell and antibody responses, and the effects that these concepts have on vaccine-development endeavors.
\end{abstract}

$\mathrm{T}$ he Flavivirus genus (Flaviviridae family) includes important and broadly distributed human pathogens such as dengue virus (DENV), Zika virus (ZIKV), yellow fever virus (YFV), Japanese encephalitis virus (JEV), West Nile virus (WNV) and tickborne encephalitis virus (TBEV). Except for TBEV, which is transmitted by ticks, these viruses are transmitted by hematophagous mosquito vectors and can be divided into those associated with neurological disease (including JEV, TBEV and WNV) and those with a tendency to cause viscerotrophic and hemorrhagic disease (YFV, DENV and ZIKV) ${ }^{1}$. Recently, ZIKV has also been associated with the development of Guillain-Barré syndrome and severe fetal malformations, along with sexual and transplacental transmission in humans, which are unique features among flaviviruses reported to date 2 .

Flaviviruses pose a major health and socioeconomic burden to endemic countries, and more than half of the global population is at direct risk of infection. In fact, estimates suggest that $\sim 400$ million cases of DENV infections occur annually, 100 million of which develop symptoms ${ }^{3}$. In addition, concerns about the potential introduction of these pathogens into new environments, together with the severe nature of the diseases, have made the development of safe and effective vaccines a top priority. In this Review, we provide a broad overview of the adaptive immune response against flavivirus infections, identify gaps in the understanding of these diseases and suggest future research priorities, with a particular emphasis on DENV and ZIKV.

\section{Structure and life cycle of flaviviruses}

Flaviviruses are enveloped viruses with a positive-sense, single-stranded RNA genome of $\sim 11 \mathrm{~kb}$. The DENV serogroup is formed by four closely related serotypes, which differ by $30-35 \%$ in amino acid identity (DENV1, 2, 3 and 4); however, strains of most individual flaviviruses show less genetic diversity and exist as a single serotype ${ }^{4}$. All flaviviruses share the same basic genomic organization and particle structure. The RNA genome encodes a precursor polyprotein $\sim 3,500$ amino acids long that is cleaved into ten polypeptides: three structural proteins (the capsid, precursor membrane (prM) and envelope glycoprotein (E)) that form the viral particle and seven nonstructural (NS) proteins (NS1, NS2A, NS2B, NS3, NS4A, NS4B and NS5) that fulfill several roles during the viral life cycle ${ }^{4}$ (Fig. 1a). The E glycoprotein is the principal constituent of the viral particle, and it has three structural domains: EDI, EDII, which carries the highly hydrophobic fusion loop required to initiate infection, and EDIII, which contains the putative receptor-biding $\operatorname{sites}^{5}$ (Fig. 1b). Fully mature particles have a relatively round, smooth surface formed by 180 copies of the E protein arranged into antiparallel homodimers that cover most of the viral surface in a herringbone pattern ${ }^{6}$ (Fig. 1c-f).

After viral entry into host cells, the acidification of the early endosome reorients the antiparallel $\mathrm{E}$ dimers into vertical parallel trimers that expose the fusion loop and mediate fusion of the viral and endosome membranes, thus releasing the viral nucleocapsid into the cytosol, where translation and replication occur (Fig. 2). Viral assembly begins when the nucleocapsid buds into the endoplasmic reticulum lumen, thereby creating immature, noninfective particles in which prM and E form spike-like trimers ( $\mathrm{prM}_{3} \mathrm{E}_{3}$ heterohexamers) across the viral surface. Exposure to low $\mathrm{pH}$ in the trans-Golgi network causes a shift toward the mature antiparallel-dimeric herringbone arrangement $\left(\operatorname{prM}_{2} \mathrm{E}_{2}\right)$, thus enabling furin protease-mediated cleavage of prM, which renders the particle mature and infective $\left(\mathrm{M}_{2} \mathrm{E}_{2}\right)$ (Fig. 3a). Once cleaved, pr remains bound to the $\mathrm{E}$ dimers, covering the hydrophobic fusion loop and thereby preventing premature viral membrane fusion with the host cell, and it dissociates after the virus is released from the infected cell, thus yielding an infectious viral particle ${ }^{7,8}$.

\section{Structural heterogeneity of flaviviruses}

The antibody response against flaviviruses is affected by the structural heterogeneity of the viral particles, which arises from several sources. Particularly in DENV, prM cleavage is inefficient and leads to the release of not only mature $\mathrm{M}_{2} \mathrm{E}_{2}$ ('smooth', fully cleaved prM) and immature $\operatorname{prM}_{3} \mathrm{E}_{3}$ ('spiky', with no prM cleavage) particles (Fig. 3a) but also a wide variety of partially mature virions, in which the surface structure is a mixture of mature and immature regions ${ }^{9,10}$ (Fig. 3b). Moreover, the efficiency of prM cleavage varies among cell types: viral particles derived from insect cells or tumor cell lines such as Vero have higher prM content than those obtained from primary human dendritic cells ${ }^{11,12}$.

The mature viral particle is a highly dynamic structure in which the E protein undergoes constant conformational changes

'Nuffield Department of Medicine, Wellcome Trust Centre for Human Genetics, University of Oxford, Oxford, UK. ${ }^{2}$ Dengue Hemorrhagic Fever Research Unit, Office for Research and Development, Siriraj Hospital, Faculty of Medicine, Mahidol University, Bangkok, Thailand. ${ }^{3}$ Division of Medical Sciences, John Radcliffe Hospital, University of Oxford, Oxford, UK. *e-mail: gavin.screaton@medsci.ox.ac.uk 
a

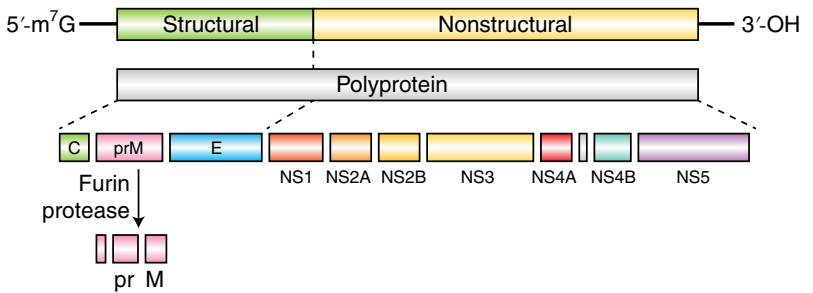

b c

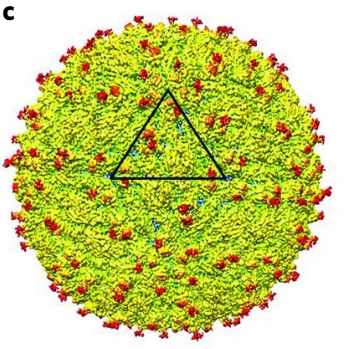

e

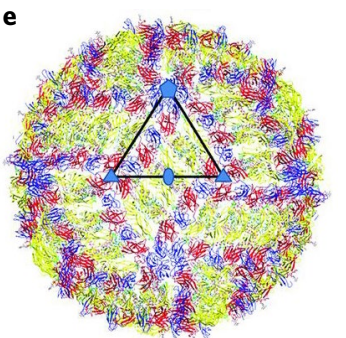

d

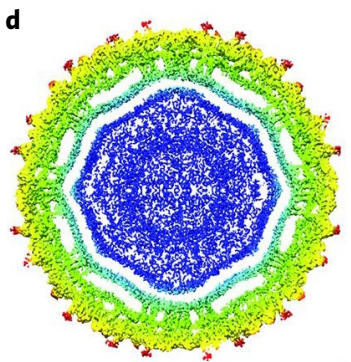

f

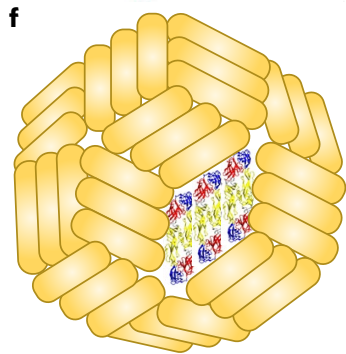

Fig. 1 | Flavivirus genomic organization and structure. a, Schematic representation of flavivirus genome organization and processing of the polypeptide into mature viral proteins ${ }^{1}$. b. Top, primary structure of DENV E-protein ectodomain showing EDI (red), EDII (yellow) and EDIII (blue). Bottom, ribbon and molecular structure of DENV E antiparallel dimer (top view, PDB 1UZG). c, Surface representation of ZIKV. The asymmetrical unit is indicated by the black triangle. Blue, up to $130 \AA$; cyan, up to $150 \AA$; green, up to $190 \AA$; yellow, up to $230 \AA$; red, above $231 \AA$. d, Depth-based, color-coded cross-sectional view of ZIKV, with color coding as in c. e, E- and M-protein $\mathrm{C} \alpha$ backbone structure on the mature ZIKV virion, highlighting the herringbone organization of $\mathrm{E}$ dimers (color coding as in $\mathbf{b}$ ). f, Schematic representation of the mature ZIKV, as shown in e. The structures shown in c-e are reprinted with permission from ref. ${ }^{6}$, AAAS.

with different thermodynamic stabilities. This 'viral breathing' provides substantial flexibility to the viral surface and varies the antigenic landscape of the virion by continuously changing accessibility to particular epitopes, thus explaining why some antibodies show enhanced binding and neutralization after prolonged incubation or incubation at elevated temperatures ${ }^{13}$. Similarly, mature virions, particularly DENV2, expand at temperatures above $33{ }^{\circ} \mathrm{C}$, acquiring a 'bumpy' appearance in which the interaction between $\mathrm{E}$ dimers changes, thus exposing otherwise occluded $\mathrm{E}$ regions ${ }^{14}$. The antibody response is also influenced by the $T=3$ quasi-icosahedral symmetry of the E protein on the viral surface; this symmetry changes epitope accessibility as a result of the different chemical environments at the two-, threeand five-fold axis ${ }^{15}$.

\section{T cell responses against flaviviruses}

Much work has been performed to identify the immunodominant epitopes recognized by $\mathrm{CD} 8^{+}$and $\mathrm{CD} 4^{+} \mathrm{T}$ cells, particularly in DENV infection. In DENV-infected patients and in volunteers vaccinated with a live attenuated tetravalent vaccine, $\mathrm{T}$ cell epitopes have been mapped to most of the DENV proteome, although $\mathrm{CD}^{+} \mathrm{T}$ cells preferentially target DENV NS3, NS5, and NS4b, whereas $\mathrm{CD} 4^{+} \mathrm{T}$ responses tend toward structural proteins and NS1 (refs. ${ }^{16-19}$ ). Similar differential targeting of $\mathrm{CD}^{+}$and $\mathrm{CD}^{+}$ $\mathrm{T}$ cells has also been observed in response to JEV infection $^{20}$ and the YFV vaccine ${ }^{21,22}$. In ZIKV-infected patients, $\mathrm{T}$ cell responses to the complete viral proteome have been reported: $\mathrm{CD}^{+} \mathrm{T}$ cells preferentially focus on structural proteins, whereas $\mathrm{CD} 4^{+} \mathrm{T}$ cells target structural and NS proteins in equal proportions, although the $\mathrm{CD}^{+} \mathrm{T}$ cell response is modulated toward NS proteins in the context of previous DENV exposure ${ }^{23}$. A separate study in a ZIKV-infected, flavivirusnaïve traveler has shown a preferential $\mathrm{CD}^{+}$and $\mathrm{CD}^{+} \mathrm{T}$ cell response toward NS2A and E proteins, respectively ${ }^{24}$.

Mouse models have been fundamental not only in determining the epitopes recognized by $\mathrm{T}$ cells but also in understanding their role during flavivirus infections. In AG129 (Ifnar1 ${ }^{-/-}$, Ifngr ${ }^{-/-}$) and human MHC class II transgenic mice, $\mathrm{CD} 4^{+} \mathrm{T}$ cell responses against NS1, NS3, NS5 and E have been observed after immunization with ZIKV recombinant proteins ${ }^{25}$, whereas similar $\mathrm{CD}^{+} \mathrm{T}$ cell profiles have been reported in infected $\mathrm{CD} 57 \mathrm{BL} / 6 \mathrm{mice}^{26}$. $\mathrm{CD} 8^{+} \mathrm{T}$ cells play a role in viral clearance from neural tissues ${ }^{27-30}$. Furthermore, $\mathrm{CD} 8^{+}$ $\mathrm{T}$ cell depletion leads to enhanced ZIKV and DENV infection, but this effect is reversed after adoptive transfer of memory $\mathrm{CD}^{+}$ $\mathrm{T}$ cells $\mathrm{s}^{26,31}$. Similar conclusions have been found by using mice deficient in various cytotoxic effector molecules in WNV infection ${ }^{27,28}$, whereas $\mathrm{CD}^{+} \mathrm{T}$ cells have been shown to be essential for controlling YFV and ZIKV infection in mice lacking a humoral response ${ }^{32,33}$. In contrast, exacerbated $\mathrm{CD}^{+} \mathrm{T}$ cell infiltration in response to $\mathrm{JEV}$, WNV and ZIKV infection has also been shown to induce cytotoxic tissue damage and to contribute to neuropathogenesis in mice ${ }^{34,35}$. In the case of $\mathrm{CD}^{+} \mathrm{T}$ cells, their role in assisting immune responses has been demonstrated in WNV-infected mice, in which both antibody and $\mathrm{CD}^{+} \mathrm{T}$ cell responses are impaired at late stages of infection in the absence of functional $\mathrm{CD} 4^{+} \mathrm{T}$ cells ${ }^{36}$, whereas viral clearance is enhanced in DENV-infected mice after vaccination with $\mathrm{CD}^{+} \mathrm{T}$ cell-specific epitopes ${ }^{37}$. Other studies have suggested that $\mathrm{CD}^{+} \mathrm{T}$ cell-mediated protection may also come from direct cytotoxic functions: WNV-infected Rag1 $^{-/-}$mice show significantly lower mortality when naïve $\mathrm{CD} 4^{+} \mathrm{T}$ cells are transferred before infection $^{38}$, whereas mice injected with JEV-primed $\mathrm{CD}^{+} \mathrm{T}$ cells are protected from infection ${ }^{39}$.

Data gathered from human subjects, although not as abundant as data from animal studies, suggest that $\mathrm{CD} 8^{+} \mathrm{T}$ cell-mediated protection from severe dengue disease is linked to production of the cytokine IFN- $\gamma$ and human leukocyte antigen (HLA) alleles that induce polyfunctional responses ${ }^{40,41}$, whereas polyfunctional $\mathrm{CD}^{+} \mathrm{T}$ cell responses with strong cytokine production correlate with limited dengue disease ${ }^{41}$ and good recovery from JEV infection $^{20}$. Further studies have suggested that DENV-specific CD $4^{+}$ $\mathrm{T}$ cells show a strong cytotoxic phenotype associated with protective immunity in infected patients ${ }^{42}$, whereas early activation and 


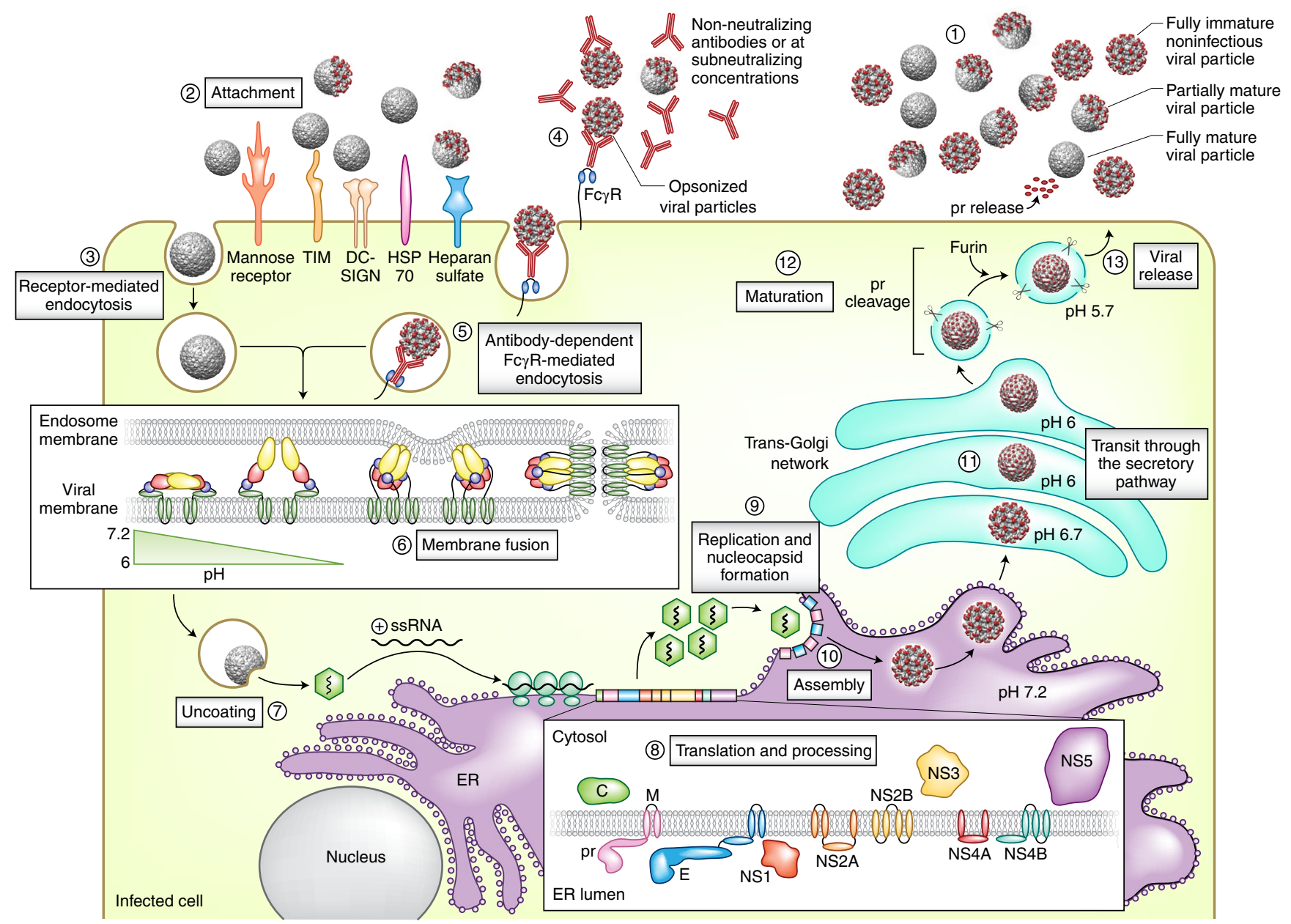

Fig. 2 | Flavivirus life cycle. (1) Flavivirus particles exist as a mixture of fully mature, partially mature and fully immature structures, depending on the extent of pr cleavage. The virus binds to surfaces of susceptible cells through interaction with attachment factors (2), and this binding is followed by interactions with secondary receptors that mediate internalization via endocytosis (3). Alternatively, in the presence of non-neutralizing antibodies (4), opsonized viral particles can be internalized via Fc $\gamma$-receptor-mediated endocytosis (5). Endosome acidification triggers $E$ trimerization and fusion of the virus and endosome membranes (6), thereby releasing the nucleocapsid into the cytosol (7). The viral genome is then translated and processed before replication commences (8). Nucleocapsids are formed (9), and assembly is initiated by budding into the endoplasmic reticulum (ER) lumen (10). The immature viruses follow the secretory pathway (11), in which the decreasing $\mathrm{pH}$ of the trans-Golgi network triggers maturation through furin proteasemediated cleavage of prM (12). Nascent virions are then secreted from the cell, and pr is released from the viral surface (13). The molecular structures showing mature, partially mature and immature viral particles were adapted with permission from ref. ${ }^{9}$, European Molecular Biology Organization. TIM, T cell immunoglobulin mucin; DC-SIGN, dendritic-cell-specific intracellular adhesion molecule-3-grabbing non-integrin; HSP, heat-shock protein; ssRNA, single-stranded RNA; C, capsid protein; $M$, membrane protein.

recruitment of IFN- $\gamma$-producing $\mathrm{CD}^{+}{ }^{+} \mathrm{T}$ cells is correlated with high titers of neutralizing antibodies (nAbs) in YFV-vaccinated subjects ${ }^{43}$. During infection with ZIKV, compared with DENV, fewer IFN- $\gamma$-producing $\mathrm{CD} 4^{+} \mathrm{T}$ cells are present, but their role remains mostly unknown ${ }^{44}$. In humans, similarly to animal models, excessive T cell infiltration may lead to immunopathogenesis; indeed, cytotoxic damage mediated by both $\mathrm{CD}^{+}$and $\mathrm{CD} 8^{+}$ $\mathrm{T}$ cells has been described in infiltrated tissues of YFV-infected patients ${ }^{45}$, and the magnitude of the $\mathrm{T}$ cell response is correlated with the severity of DENV infection ${ }^{16,46}$. In addition, direct cytolysis of bystander cells by DENV-specific $\mathrm{CD}^{+} \mathrm{T}$ cells in vitro suggests that $\mathrm{T}$ cell infiltration may be a potential cause of liver damage during infection ${ }^{47}$. In another study, $\mathrm{T}$ cells expressing low levels of CD107a (a marker of cytotoxic activity) and high levels of cytokines have been found to be strongly associated with severe disease during primary and secondary DENV infection in children, whereas high CD107a expression is correlated with better outcomes after infection ${ }^{16}$, thus suggesting that both the magnitude and the functional phenotype of the anti-DENV T cell response are associated with disease severity.

These data indicate that the amplitude of the $T$ cell response plays a role in determining the severity of disease after flavivirus infection: whereas cytokine production is fundamental in coordinating the different elements of the immune response, $\mathrm{CD} 4^{+}$and $\mathrm{CD} 8^{+}$ $\mathrm{T}$ cell-mediated cytotoxicity appears to be required for early containment of the pathogen; however, excessive cytotoxicity might lead to tissue damage in infiltrated organs, whereas excessive production of proinflammatory cytokines might lead to increased disease severity in the context of high viral/antigenic loads.

\section{Cross-reactive immunity and T cell original antigenic sin}

The high degree of sequence identity shared among flaviviruses results in cross-reactive responses that can act as a 'double-edged sword, either boosting protection or compromising the immune response after sequential infection. In sequential infections with viruses of related sequence, the secondary response, involving 


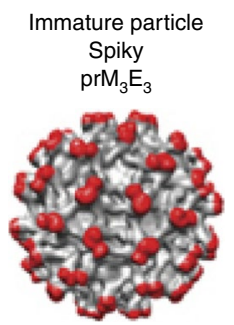

$$
\begin{gathered}
\text { Immature particle } \\
\text { Smooth }
\end{gathered}
$$
$\mathrm{prM}_{2} \mathrm{E}_{2}$
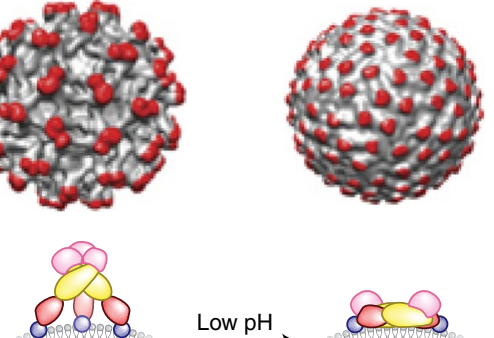
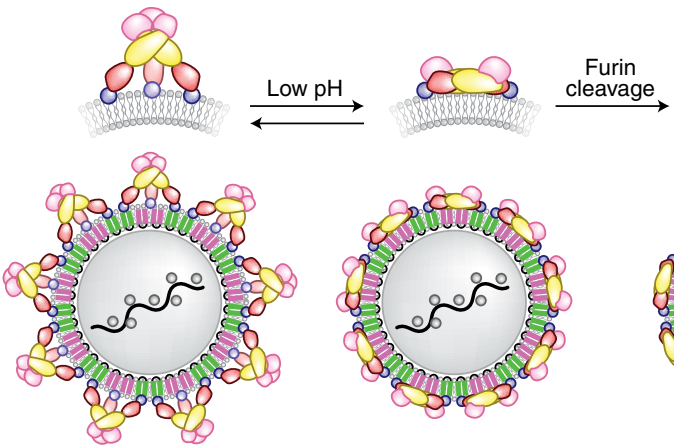
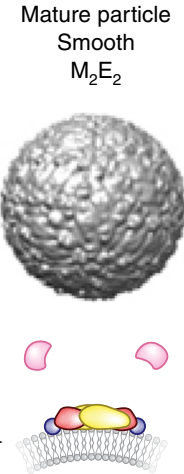

$\mathrm{M}_{2} \mathrm{E}_{2}$
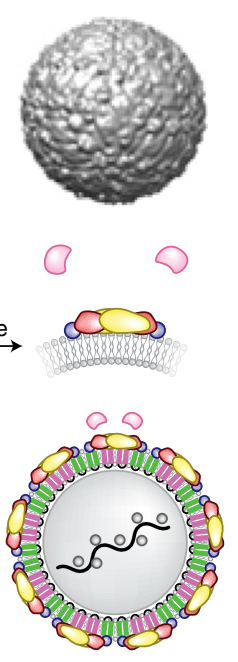

b
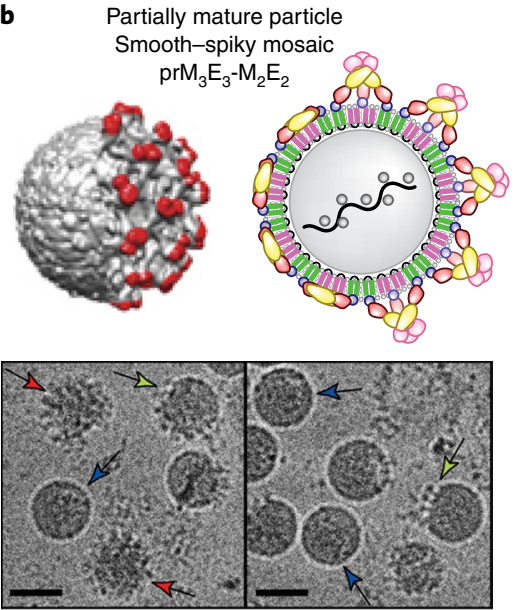

Fig. 3 | Structural heterogeneity of flavivirus particles during maturation. a, Left, cryo-EM reconstruction and schematic representation of the immature virion at neutral $\mathrm{pH}$ after budding into the endoplasmic reticulum, showing the $\mathrm{prM}_{3} \mathrm{E}_{3}$ 'spiky' surface arrangement. Middle, low-pH exposure induces a $\operatorname{prM}_{2} \mathrm{E}_{2}$ flat dimeric conformation on the immature particle. Right, the mature $\mathrm{M}_{2} \mathrm{E}_{2}$ 'smooth' dimeric structure after furin protease-mediated cleavage of pr and release of the particle into the extracellular milieu ${ }^{715}$. b. Top, Cryo-EM reconstruction and schematic representation of a partially mature particle, showing a mixture of smooth and spiky surfaces as a result of incomplete pr cleavage. Bottom, cryo-EM images of two WNV preparations. Red, blue and green arrows indicate fully mature, fully immature and partially mature mosaic particles, respectively. Scale bars, $500 \AA$. As in Fig. 1e, domains I, II and III are shown in red, yellow and blue, respectively; prM (pink) is also shown. The molecular models and the cryo-EM image were adapted with permission from ref. ${ }^{9}$ and ref. ${ }^{10}$, European Molecular Biology Organization, respectively.

either antibodies or $\mathrm{T}$ cells, can be dominated by recall of memory cells generated during the primary exposure. This process was first described for influenza antibody responses, and because the primary and secondary infecting viruses may differ substantially, the recall of a memory response may lead to mobilization of low-avidity clones, which may in turn compromise the response to the secondary infection, a process termed original antigenic $\sin ^{48}$.

The association of severe dengue symptoms of vascular leakage with steep decreases in viral load, T cell activation and a 'storm' of proinflammatory cytokines suggests that $\mathrm{T}$ cells may induce immunopathology in severe disease. Many cross-reactive memory $\mathrm{T}$ cells are mobilized during secondary flavivirus infection, and these cells frequently have higher avidity to the primary infecting virus and show skewed responses with more cytokine production and less degranulation ${ }^{16,46,49}$. Indeed, the magnitude of the anti-DENV T cell response correlates with disease severity after secondary DENV infection in humans ${ }^{46}$. An interesting new immunocompetent mouse model has recently been described, in which C57BL/6 mice were sequentially infected with DENV1 followed by DENV2 and compared with mice receiving either placebo followed by DENV2, or DENV2 followed by DENV2. The mice challenged with DENV1 followed by DENV2 showed significant increases in liver enzymes and longer bleeding times than did mice challenged with placebo followed by DENV2, or DENV2 followed by DENV2. Adoptive transfer of DENV1-specific CD8 ${ }^{+} \mathrm{T}$ cells before DENV2 infection is necessary and sufficient to induce manifestations of severe disease in this immunocompetent mouse model ${ }^{50}$.

Although cross-reactive $\mathrm{T}$ cell responses have the potential to promote immunopathology, they can also be protective. $\mathrm{T}$ cell responses against ZIKV infection have been shown to be more rapidly mobilized in the presence of preexisting DENV immunity than in the presence of ZIKV infection in DENV-naïve patients, although the overall epitope landscape across the ZIKV and DENV proteomes differs, and it is unclear whether these cross-reactive responses would be protective or, through original antigenic sin, would exacerbate ZIKV infection ${ }^{23}$. In mice, DENV-specific CD8 ${ }^{+}$ $\mathrm{T}$ cells have been shown to mediate protection against other heterologous DENV serotypes and ZIKV, despite having an altered phenotype ${ }^{51-53}$. Similarly, JEV-vaccinated mice are protected against all DENV serotypes ${ }^{54}$. Notably, almost all animal T cell studies have been performed in immunocompromised mice, which do not recapitulate the human clinical syndrome in which peaks of illness coincide with steep decreases in viral load.

The role of T cells in DENV pathogenesis remains controversial, and it is frequently polarized between protection and immunopathology. In our view, in different individuals these cells may play protective or pathogenic roles, which may be determined by the sequence of infection, the degree of cross-reactivity, the number and function of the T cells, and the viral load that they confront. Further studies are needed to fully determine the role of $\mathrm{T}$ cell responses in flavivirus infections and how cross-reactive responses influence the outcomes of subsequent infections.

\section{The antibody response against flaviviruses}

Development of nAbs is widely considered crucial for immune responses to viral infections. In the case of flaviviruses, the $\mathrm{E}$ protein, prM and NS1 are the main targets of the antibody response ${ }^{15}$. Antibody-mediated neutralization of flaviviruses follows a 'multihit' model that depends on a critical number of antibody molecules being bound to the virion; this threshold depends on antibody affinity and epitope accessibility, the latter of which is heavily influenced by structural heterogeneity and explains why strongly neutralizing antibodies usually target highly accessible epitopes, whereas poorly neutralizing antibodies tend to bind cryptic epitopes. Because exposed surfaces show the highest degree of variation among flaviviruses, most potent neutralizing monoclonal antibodies (mAbs) are type specific, whereas antibodies promoting antibody-dependent enhancement of infection (ADE) tend to target highly cross-reactive epitopes ${ }^{15}$. 

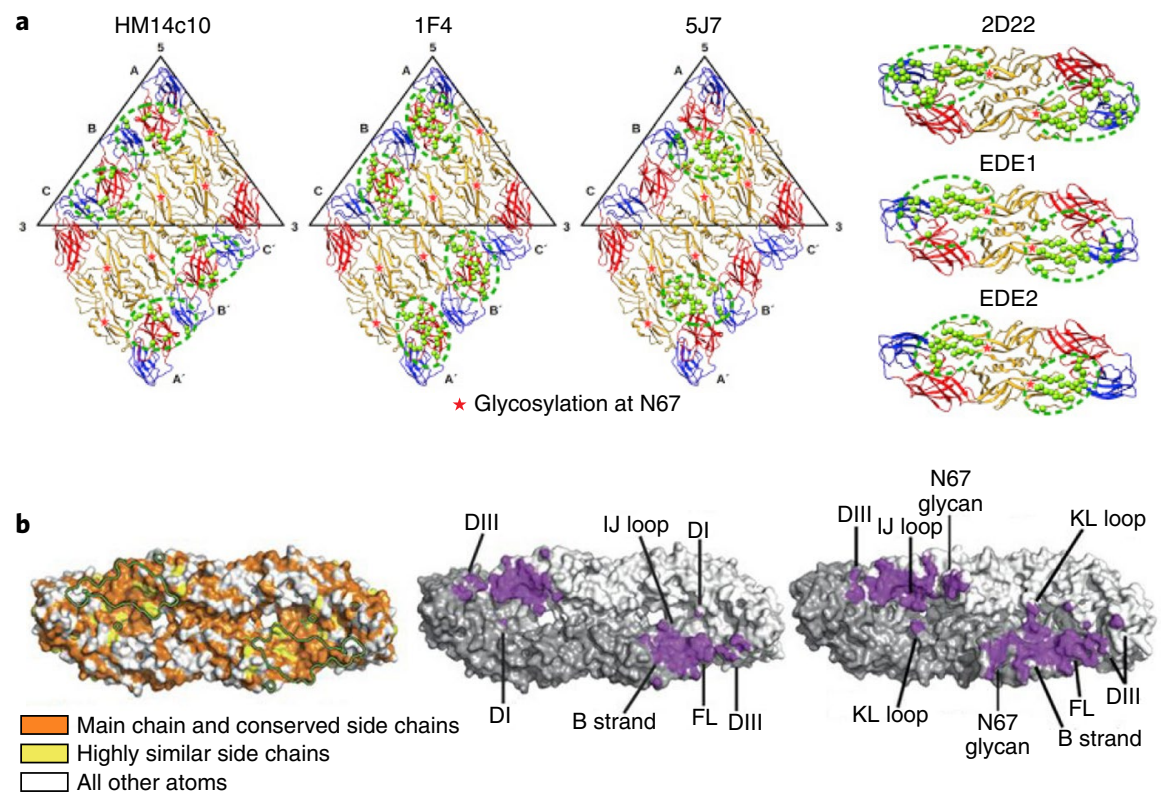

Fig. 4 | Complex quaternary epitopes on the viral surface. a, Dimer- and higher-structural-order-dependent quaternary epitopes bound by some highly neutralizing flavivirus $m A b s$. The green spheres show the epitope residues, and the approximate footprints of the mAbs are marked by a dotted green line. b, Left, top view of the ZIKV E dimer showing the degree of amino acid conservation between DENV and ZIKV and the outline of an EDE1 mAb (EDE1-C8) footprint. Middle and right, EDE1-C8 mAb footprint on ZIKV (middle) and DENV (right) E dimers. DI and DIII indicate E domains I and III respectively. Figure adapted with permission from ref. ${ }^{64}$, Elsevier, and ref. ${ }^{75}$, Springer Nature.

Although neutralizing epitopes have been described on all three structural domains of $\mathrm{E}$, mAbs mapped to the highly variable EDIII frequently have potent neutralizing activity ${ }^{55,56}$. Surprisingly, whereas antibodies to EDIII are a major component of the antibody response in mice, they are only a small component of the human response against DENV and $\mathrm{WNV}^{57,58}$, and they do not contribute significantly to the neutralization activity present in serum ${ }^{59}$. In contrast, the high anti-EDIII titers and EDIII-specific neutralizing activity found in ZIKV-infected patients suggest that antibodies to EDIII are more important in ZIKV immunity ${ }^{60,61}$. Interestingly, some of these antibodies against ZIKV-EDIII cross-react with DENV and may have been generated from memory cells in previously DENV1-infected patients ${ }^{60}$.

Antibodies targeting the fusion-loop epitope (FLE) are a major immunodominant component of the humoral response against flaviviruses $^{55,57,58}$. The FLE is normally hidden at the E-dimer interface; however, its exposure is substantially increased by the presence of prM in immature patches on the viral surface and by the effect of viral breathing ${ }^{10,62}$. In contrast to anti-EDIII, anti-FLE mAbs are highly cross-reactive and show poor neutralizing properties, particularly on viruses containing low levels of $\mathrm{prM}^{12,63}$.

The symmetric distribution of $\mathrm{E}$ on the virus surface creates complex quaternary and conformational epitopes that are present only on the intact virion and are targeted by some of the most potent $\mathrm{nAbs}$ found in samples from DENV-immune patients ${ }^{12,64-66}$ (Fig. 4a). Some of these epitopes are defined by the herringbone arrangement of $\mathrm{E}$ and are thus restricted to the viral surface: the epitope of mAb HM14c10 involves two adjacent DENV1 E dimers ${ }^{65}$, and $\mathrm{mAb} 5 \mathrm{~J} 7$ has critical interactions with three adjacent DENV3 E monomers ${ }^{67}$, whereas DENV1-specific mAb 1F4 targets an epitope restricted to a single $\mathrm{E}$ monomer but on a particular conformation that exists only on the virus ${ }^{68}$. A different group of quaternary epitopes are restricted to the E-E dimer interface and do not require higher-order arrangements of the protein. The mAb 2D22, for example, binds the DENV2 E-dimer interface and has substantial interactions with EDIII residues ${ }^{69}$. Similar antibodies have also been described in WNV-, JEV- and ZIKV-infected patients ${ }^{70-72}$.
A group of potent neutralizing human mAbs isolated from DENV-infected patients recognize a highly conserved epitope, the E-dimer epitope (EDE), which spans the valley formed at the EDI/ DIII-EDII interface of the $\mathrm{E}$ dimer, which also contains the fusion loop. The EDE overlaps with the binding site of prM with $\mathrm{E}$ dimers, thus also potentially explaining why the sequence of the epitope is so highly conserved between DENV and ZIKV ${ }^{12,73}$ (Fig. 4b), and why antibody-escape mutations do not readily develop ${ }^{74}$.

Antibodies to EDE are further classified into two groups on the basis of the requirement of E glycosylation for binding: anti-EDE1 $\mathrm{mAbs}$ are not affected by N153 glycosylation, whereas anti-EDE2 mAbs show better binding in presence of the glycan ${ }^{73}$. Both antiEDE1 and anti-EDE2 mAbs potently neutralize (down to low-picomolar levels) all four DENV serotypes ${ }^{12}$, whereas anti-EDE1 can also potently neutralize $\mathrm{ZIKV}^{62,75}$. Binding of anti-EDE2 mAbs to ZIKV is significantly lower than binding to DENV, owing to a change in the orientation of the glycosylation motif ${ }^{5}$. Antibodies to EDE, unlike those to FLE, are not dependent on the level of prM present on virions; testing of a panel of anti-FLE and anti-EDE mAbs on DENV viruses made in insect cells or primary dendritic cells has shown that only the anti-EDE mAbs fully neutralize dendritic cell virus (with low prM content), whereas both anti-FLE and anti-EDE mAbs fully neutralize insect virus (with high prM content) ${ }^{12}$.

Antibodies to prM have been described in DENV- and WNVinfected patients; in DENV infection, anti-prM-producing cells make up a substantial portion of the anti-DENV memory B cell response, and most anti-prM antibodies are fully cross-reactive among the four DENV serotypes ${ }^{11,66,76}$. Anti-prM antibodies show poor neutralizing ability in vitro and in vivo, which reaches a plateau at approximately $50 \%$ neutralization, presumably because many virions either lack prM altogether and cannot bind anti-prM antibodies or contain too few prM molecules to allow for neutralization ${ }^{11,66,76}$.

As detailed above, the cleavage of prM is frequently incomplete in DENV infection, thus leading to the production of partially mature virus particles containing variable amounts of cleaved and uncleaved prM. Studies of the furin-cleavage site at the $\mathrm{pr}-\mathrm{M}$ junction have indicated that this region has a suboptimal sequence 
compared with the consensus; indeed, a single amino acid mutation leading to an E-to-A change at the P3 position (RREKR to RRAKR after the pr E203A mutation, with the change shown in boldface) leads to a $36 \%$ increase in prM cleavage in the DENV2 strain 16681 (ref. ${ }^{77}$ ). The maintenance of this suboptimal cleavage site in DENV may indicate a selective pressure to maintain high prM content to drive an immunological evasion mechanism exploited by the virus, i.e., the generation of two suboptimal yet dominant antibody responses to prM and to the FLE, which are poorly neutralizing and drive ADE (described below).

Beyond antibodies to E and prM, antibodies to NS1 can also be readily detected after a flavivirus infection. The flavivirus NS1 protein exists in multiple oligomeric forms: intracellular monomers have important roles during viral replication, whereas hydrophobic dimers are usually attached to membranes of infected cells, and high levels of a lipid-cored hexameric secretory form are found in patient sera during acute infection ${ }^{78}$.

Recent reports have suggested that DENV NS1 directly promotes vascular permeability by inducing a strong proinflammatory vasoactive response via Toll-like receptor 4 signaling ${ }^{79}$ and endothelial glycocalyx disruption ${ }^{80}$. Early studies have demonstrated that passive immunization with anti-NS1 mAbs protects mice against lethal YFV and DENV2 challenge, an effect possibly dependent on complement-mediated lysis of infected cells ${ }^{81,82}$, whereas anti-NS1-mediated antibody-dependent cytotoxicity contributes to protection against WNV infection in mice $^{83}$. In addition, polyclonal sera from DENV NS1-immunized mice and anti-NS1 mAbs have been shown to prevent vascular leakage and to protect against lethal DENV2 challenge ${ }^{84}$. In contrast, antibodies to DENV NS1 have been proposed to participate in severe DENV pathogenesis by promoting bleeding diathesis through inhibition of thrombin activity and enhancement of fibrinolysis ${ }^{85,86}$. However, this finding seems to be in conflict with disease dynamics in humans, because recovery from severe dengue pathogenesis occurs while these antibodies are still in circulation.

\section{Antibody-dependent enhancement and cross-reactive- antibody responses}

The ADE hypothesis was proposed to explain the increase in dengue pathogenicity seen in secondary DENV infections and primary infections in infants born to dengue-immune mothers ${ }^{87,88}$. After a primary infection, an individual will have a neutralizing typespecific response. After infection on a second occasion with a virus whose E-protein sequence differs by $30-35 \%$, cross-reactive antibodies to the primary virus may not be present at sufficient concentration or avidity to neutralize the second virus yet may still be able to bind and opsonize viral particles. Opsonized virus particles can be internalized into myeloid cells by Fc $\gamma$ receptor-mediated endocytosis, thus driving higher viral replication and peak viral loads. $\mathrm{ADE}$ is readily demonstrated in both in vitro and in vivo models, and almost any antibody present at subneutralizing concentrations has the potential to induce $\mathrm{ADE}^{89}$.

The demonstration that the increased risk of dengue hemorrhagic fever/dengue shock syndrome (DHF/DSS) during the first year of life in infected infants born to DENV-immune mothers correlates with the decline in maternally acquired antibodies is among the most compelling evidence for ADE-mediated pathogenesis ${ }^{90}$. A recent study in a cohort of Nicaraguan children has correlated low antibody titers from previous DENV immunity with an increased risk of DHF/DSS, thus implying that cross-reactive antibodies that also have poor neutralizing ability are required to promote ADE in humans ${ }^{91}$. Finally, the increase in hospitalization from symptomatic dengue in young children given the Dengvaxia vaccine, compared with control vaccines ${ }^{92}$, is believed by some to be the result of the vaccine priming but not protecting dengue-naïve vaccinees, thereby promoting $\mathrm{ADE}$ after natural infection ${ }^{93,94}$.
Inefficient prM cleavage, described above as a potential immunological evasion strategy, appears to promote the generation of ADE-inducing antibodies: studies have suggested that antibodies targeting prM and FLE, which are poorly neutralizing, are the main serum component responsible for ADE in vivo and in vitro ${ }^{95}$. AntiprM and anti-FLE antibodies can also endow viruses with very high prM content (which are themselves not infectious) with the ability to infect myeloid cells. This ability is achieved through promotion of Fcy receptor-mediated uptake and subsequent prM cleavage and maturation, thus enabling the virus to fuse and productively infect cells ${ }^{11,76}$. An alternative ADE mechanism independent of Fcy receptor interaction has been described recently, in which antibody binding to the viral particle induces structural rearrangements of $\mathrm{E}$ that expose the fusion-loop-enhancing infection with $\mathrm{TBEV}^{96}$. The involvement of T cell responses in the context of ADE has been studied. $\mathrm{CD}^{+} \mathrm{T}$ cells prevent ADE-mediated lethality in DENV-challenged mice ${ }^{97}$ and provide protection against heterotypic DENV infection despite the presence of subneutralizing titers of cross-reactive antibodies in the Ifnar1 ${ }^{-/-}$(A129) mouse model ${ }^{52}$.

The potential for cross-reactive-antibody responses to promote $\mathrm{ADE}$ has gained some prominence after the recent ZIKV outbreak, which occurred in areas with high DENV exposure, thus suggesting that anti-DENV immunity might promote ADE of ZIKV and vice versa. Early studies have demonstrated an enhancement of DENV and ZIKV infection in vitro by antibodies against a wide variety of flaviviruses ${ }^{98,99}$. Similarly, sera from DENV- and ZIKV-infected patients can also enhance DENV and ZIKV infection in vitro ${ }^{55,62,100}$. The first in vivo evidence of immunological interaction between DENV and ZIKV came from the observation of lethal enhancement of DENV infection in AG129 mice after passive transfer of cross-reactive antibodies isolated from ZIKV- and DENV-infected patients ${ }^{55}$. In another study, enhancement of ZIKV infection has been demonstrated in Stat $2^{-/-}$ mice after intraperitoneal administration of DENV- or WNVimmune sera ${ }^{101}$. Evidence obtained from nonhuman primates is contrasting: a recent study has shown enhancement of DENV2 infection in ZIKV-immune macaques ${ }^{102}$, whereas another report has shown that previous DENV or YFV exposure has no effect on ZIKV infection, as compared with the response in naïve nonhuman primates ${ }^{103}$. Furthermore, a recent study in ZIKV-infected pregnant women has found that antibodies to DENV at the time of infection are not significantly associated with congenital Zika syndrome $e^{104}$. In other human studies, cross-reactive antibodies induced after JEV vaccination have been suggested to be associated with an increased risk of symptomatic dengue illness ${ }^{105,106}$ and prolonged viremia of the attenuated YFV vaccine ${ }^{107}$.

Whereas clinical and experimental data strongly support the role of $\mathrm{ADE}$ in the pathogenesis of DENV, DENV/ZIKV crossreaction in ZIKV infection and cross-placental transfer warrants further study, which is currently constrained by the difficulty of disentangling infection histories serologically in large epidemiological cohorts.

\section{Flavivirus vaccines}

The history of flavivirus-vaccine development is mixed: although successful vaccines against YFV and JEV and TBEV are available, human WNV vaccines are lacking, and the only licensed DENV vaccine, Sanofi-Pasteur's Dengvaxia (CYD-TDV), has substantial limitations. In response to the ZIKV epidemic, a series of new vaccine candidates are being developed and tested. Licensed vaccines and candidates undergoing clinical trials have been recently reviewed in detail ${ }^{108}$. The need to generate balanced, long-lasting neutralizing responses against all four serotypes of DENV to avoid the risk of $\mathrm{ADE}$ is formidable. The challenge is made more difficult by the lack of immunocompetent animal models of dengue infection and pathogenesis, and the current lack of robust human 
immune correlates of protection. To address this deficiency, human DENV-challenge models are being developed, in which potential vaccines can be tested on small numbers of volunteers before largescale clinical studies are performed ${ }^{109}$.

Dengvaxia is a tetravalent live-attenuated vaccine made of four recombinant viruses based on the backbone of the YFV-17D attenuated virus, in which the genes encoding prM and E have been substituted with those of the different DENV serotypes ${ }^{110}$. Safety and immunogenicity in humans have been demonstrated in phase I studies; however, phase II trials have revealed that the responses induced by the vaccine are not equally effective against the different DENV serotypes: there is a notable lack of efficacy against DENV2 and neutralizing titers are lower overall in this model than in animal models ${ }^{110,111}$. Although protection against DENV2 showed improvements in phase III trials, these studies have revealed that vaccine efficacy is significantly higher in DENV-immune subjects than naïve subjects ${ }^{112,113}$. Follow-up studies have shown an elevated risk of severe dengue in the 2 - to 5 -year age group ${ }^{92}$, thus suggesting that the vaccine mimics a primary DENV infection in naive patients and may potentially increase their vulnerability to $\mathrm{ADE}^{93,94}$. Indeed, in a recent 5-year efficacy and safety analysis of Dengvaxia, SanofiPasteur confirmed that vaccination of seronegative individuals led to a significant increase in the number of hospitalizations and severe dengue cases after primary DENV infection ${ }^{114}$; as a result, the vaccine is now indicated only for DENV-immune individuals older than 9 years of age in endemic countries.

The elements required to produce an ideal flavivirus vaccine are unknown, and although different approaches have been developed to assess vaccine efficacy, none have been able to provide a comprehensive measure of all the components that might be involved in protective immunity. Although definitive evidence is lacking, induction of $\mathrm{T}$ cell responses may improve the efficacy and safety of flavivirus vaccines, and future candidates will probably benefit from being rationally designed to promote activation of $\mathrm{CD}^{+}$ and $\mathrm{CD}^{+} \mathrm{T}$ lymphocytes. Such design is particularly important for vaccine platforms based on chimeric, inactivated or subunit approaches, which may poorly stimulate $\mathrm{T}$ cells because of the lack of NS-protein production ${ }^{115}$. In fact, Dengvaxia has been found not to induce $\mathrm{CD}^{+} \mathrm{T}$ cell responses in DENV-naïve individuals but to boost DENV NS3 responses in immune subjects ${ }^{116}$, thus providing a potential explanation for the poor efficacy of Dengvaxia in the DENV-naïve population. The existence of well-established correlates of protection for YFV and JEV vaccines, as well as estimates of protection for TBEV, all of which are based on measurement of $\mathrm{nAb}$ titers $(1: 10,1: 5 \text { and } 1: 10 \text {, respectively })^{117}$, clearly indicates that the quality of the antibody response is an important element in protection by flavivirus vaccines. Two further tetravalent live attenuated vaccines, produced by Takeda and NIH/Butantan, are currently in phase III trials. These vaccines are both built on a DENV backbone rather than a YFV backbone, and it is hoped that they may drive the generation of protective anti-DENV $\mathrm{T}$ cell responses and thereby perform better than Dengvaxia ${ }^{108}$.

NS1-based vaccines can lead to protective immunity: a single dose of a modified Vaccinia Ankara virus expressing ZIKV NS1 has been found to induce robust and protective cellular and humoral responses in mice ${ }^{118}$. Notably, introduction of JEV NS1 into a chimeric live JEV/WNV vaccine (RepliVAX JE, a singlecycle virus carrying JEV prME proteins on a WNV backbone) leads to a higher anti-E response and overall efficiency in mice ${ }^{119}$. Moreover, C-terminally-modified DENV NS1 antigens induced protective immune responses while preventing the induction of autoantibodies $^{120}$.

As discussed above, the potentially deleterious properties of anti-prM and anti-FLE should be considered when designing new immunogens as potential subunit vaccines. prM is essential during the viral cycle ${ }^{121}$ and is thus required in the context of live vaccines but can be removed from E-based-subunit vaccines. The critical role of the FLE in viral infection and E structure make its exclusion from vaccines far more complex. Introduction of FLE mutations (G106R and L107D) in a DENV1 E-based DNA vaccine has been found to decrease DENV2 enhancement in mice ${ }^{122}$, whereas a study on a ZIKV prME-based mRNA vaccine has shown that four FLE mutations (T76R, Q77E, W101R and L107R) diminish DENV enhancement but lead to a significant decrease in neutralizing titers when compared with wild-type vaccines (approximately sevenfold) ${ }^{123}$.

The recent demonstration that highly potent and fully crossreactive antibodies can be generated by natural DENV infection suggests that immunization strategies might be designed to favor protective cross-reactive responses and that tetravalent formulations to induce DENV-serotype-specific immunity may not be mandated. The EDE is one such cross-reactive epitope; antibodies to the EDE can neutralize all four DENV serotypes as well as ZIKV. Stable covalently linked E dimers that do not contain prM have been produced and also decrease exposure of the FLE ${ }^{124,125}$. Whether such subunit vaccines are able to induce broadly protective responses targeting quaternary epitopes remains to be determined.

\section{Concluding remarks}

The paradoxical protective or pathogenic nature of flavivirus immunity has long been recognized and is proving particularly challenging to the development of a DENV vaccine. Recent efforts examining human antibody responses have defined epitopes for potently neutralizing antibodies and have also revealed immunodominant poorly neutralizing but enhancing responses. The results of two large phase III studies of live attenuated dengue vaccines will soon be available. If these vaccines, like Dengvaxia, show suboptimal efficacy and enhance disease in naïve subjects, there will be an urgent need to explore new vaccine strategies for DENV together with defining robust correlates of protection.

Received: 10 May 2018; Accepted: 16 August 2018; Published online: 17 October 2018

\section{References}

1. Family - Flaviviridae. in Virus Taxonomy (eds. King, A.M.Q. et al.) 1003-1020 (Elsevier, San Diego, 2012).

2. Culshaw, A., Mongkolsapaya, J. \& Screaton, G. The immunology of Zika virus. F1000Res. 7, 203 (2018).

3. Bhatt, S. et al. The global distribution and burden of dengue. Nature 496, 504-507 (2013).

4. Lindenbach, B.D., Heinz-Jurgen, T. \& Rice, C.M. Flaviviridae: the viruses and their replication. in Fields' Virology 5th edn. (eds. Fields, B.N., Knipe, D.M. \& Howley, P.M.) (Wolters Kluwer Health/Lippincott Williams \& Wilkins, Philadelphia, 2007).

5. Modis, Y. Relating structure to evolution in class II viral membrane fusion proteins. Curr. Opin. Virol. 5, 34-41 (2014).

6. Sirohi, D. et al. The $3.8 \AA$ resolution cryo-EM structure of Zika virus. Science 352, 467-470 (2016).

7. Perera, R. \& Kuhn, R. J. Structural proteomics of dengue virus. Curr. Opin. Microbiol. 11, 369-377 (2008).

8. Yu, I. M. et al. Structure of the immature dengue virus at low $\mathrm{pH}$ primes proteolytic maturation. Science 319, 1834-1837 (2008).

9. Plevka, P. et al. Maturation of flaviviruses starts from one or more icosahedrally independent nucleation centres. EMBO Rep. 12, 602-606 (2011).

10. Cherrier, M. V. et al. Structural basis for the preferential recognition of immature flaviviruses by a fusion-loop antibody. EMBO J. 28, 3269-3276 (2009).

11. Dejnirattisai, W. et al. Cross-reacting antibodies enhance dengue virus infection in humans. Science 328, 745-748 (2010).

This paper shows that antibodies to prM are strong inducers of ADE and have poor neutralizing activity.

12. Dejnirattisai, W. et al. A new class of highly potent, broadly neutralizing antibodies isolated from viremic patients infected with dengue virus. Nat. Immunol. 16, 170-177 (2015).

This paper describes human mAbs against the quaternary EDE epitopes and their ability to potently neutralize all four DENV serotypes. 
13. Kuhn, R. J., Dowd, K. A., Beth Post, C. \& Pierson, T. C. Shake, rattle, and roll: Impact of the dynamics of flavivirus particles on their interactions with the host. Virology 479-480, 508-517 (2015).

14. Zhang, X. et al. Dengue structure differs at the temperatures of its human and mosquito hosts. Proc. Natl. Acad. Sci. USA 110, 6795-6799 (2013).

15. Rey, F. A., Stiasny, K., Vaney, M. C., Dellarole, M. \& Heinz, F. X. The bright and the dark side of human antibody responses to flaviviruses: lessons for vaccine design. EMBO Rep. 19, 206-224 (2018).

This review describes in detail the structural properties of the flavivirus particle, their interaction with the humoral response and the mechanisms involved in antibody-mediated neutralization.

16. Duangchinda, T. et al. Immunodominant T-cell responses to dengue virus NS3 are associated with DHF. Proc. Natl. Acad. Sci. USA 107, 16922-16927 (2010)

17. Rivino, L. et al. Differential targeting of viral components by $\mathrm{CD} 4^{+}$ versus $\mathrm{CD} 8^{+} \mathrm{T}$ lymphocytes in dengue virus infection. J. Virol. 87, 2693-2706 (2013).

18. Weiskopf, $\mathrm{D}$. et al. The human $\mathrm{CD}^{+} \mathrm{T}$ cell responses induced by a live attenuated tetravalent dengue vaccine are directed against highly conserved epitopes. J. Virol. 89, 120-128 (2015).

19. Grifoni, A. et al. Global assessment of dengue virus-specific $\mathrm{CD} 4^{+} \mathrm{T}$ cell responses in dengue-endemic areas. Front. Immunol. 8, 1309 (2017).

20. Turtle, L. et al. Human T cell responses to Japanese encephalitis virus in health and disease. J. Exp. Med. 213, 1331-1352 (2016).

21. James, E. A. et al. Yellow fever vaccination elicits broad functional CD4 $\mathrm{T}$ cell responses that recognize structural and nonstructural proteins. J. Virol. 87, 12794-12804 (2013).

22. Akondy, R. S. et al. The yellow fever virus vaccine induces a broad and polyfunctional human memory $\mathrm{CD}^{+} \mathrm{T}$ cell response. J. Immunol. 183, 7919-7930 (2009).

23. Grifoni, A. et al. Prior Dengue virus exposure shapes T cell immunity to Zika virus in humans. J. Virol. https://doi.org/10.1128/JVI.01469-17 (2017).

24. Ricciardi, M. J. et al. Ontogeny of the B- and T-cell response in a primary Zika virus infection of a dengue-naïve individual during the 2016 outbreak in Miami, FL. PLoS Negl. Trop. Dis. 11, e0006000 (2017).

25. Reynolds, C. J. et al. T cell immunity to Zika virus targets immunodominant epitopes that show cross-reactivity with other Flaviviruses. Sci. Rep. 8, 672 (2018).

26. Elong Ngono, A. et al. Mapping and role of the $\mathrm{CD} 8^{+} \mathrm{T}$ cell response during primary Zika virus infection in mice. Cell Host Microbe 21, 35-46 (2017).

27. Shrestha, B., Samuel, M. A. \& Diamond, M. S. CD8 ${ }^{+}$T cells require perforin to clear West Nile virus from infected neurons. J. Virol. 80 119-129 (2006).

28. Shrestha, B., Pinto, A. K., Green, S., Bosch, I. \& Diamond, M. S. CD8 ${ }^{+}$ T cells use TRAIL to restrict West Nile virus pathogenesis by controlling infection in neurons. J. Virol. 86, 8937-8948 (2012).

29. Jain, N. et al. CD8 T cells protect adult naive mice from JEV-induced morbidity via lytic function. PLoS Negl. Trop. Dis. 11, e0005329 (2017).

30. Huang, $\mathrm{H}$. et al. $\mathrm{CD}^{+} \mathrm{T}$ cell immune response in immunocompetent mice during Zika virus infection. J. Virol. 91, e00900-17 (2017).

31. Yauch, L. E. et al. A protective role for dengue virus-specific CD8 ${ }^{+} \mathrm{T}$ cells. J. Immunol. 182, 4865-4873 (2009).

32. Bassi, M. R. et al. $\mathrm{CD}^{+} \mathrm{T}$ cells complement antibodies in protecting against yellow fever virus. J. Immunol. 194, 1141-1153 (2015).

33. Nazerai, L. et al. A new in vivo model to study protective immunity to Zika virus infection in mice with intact type I interferon signaling. Front. Immunol. 9, 593 (2018)

34. Wang, Y., Lobigs, M., Lee, E. \& Müllbacher, A. CD8 ${ }^{+}$T cells mediate recovery and immunopathology in West Nile virus encephalitis. J. Virol. 77, 13323-13334 (2003).

35. Jurado, K. A. et al. Antiviral CD8 T cells induce Zika-virus-associated paralysis in mice. Nat. Microbiol. 3, 141-147 (2018) This paper provides evidence that $\mathrm{CD8}^{+} \mathrm{T}$ cell-derived immunopathology might be involved in the development of neural complications in ZIKV-infected mice with impaired innate resistance.

36. Phares, T. W. et al. CD4 T cells promote CD8 T cell immunity at the priming and effector site during viral encephalitis. J. Virol. 86, 2416-2427 (2012).

37. Yauch, L. E. et al. CD4 ${ }^{+} \mathrm{T}$ cells are not required for the induction of dengue virus-specific $\mathrm{CD} 8{ }^{+} \mathrm{T}$ cell or antibody responses but contribute to protection after vaccination. J. Immunol. 185, 5405-5416 (2010).

38. Brien, J. D., Uhrlaub, J. L. \& Nikolich-Zugich, J. West Nile virus-specific CD4 T cells exhibit direct antiviral cytokine secretion and cytotoxicity and are sufficient for antiviral protection. J. Immunol. 181, 8568-8575 (2008).

39. Biswas, S. M., Ayachit, V. M., Sapkal, G. N., Mahamuni, S. A. \& Gore, M. M. Japanese encephalitis virus produces a CD4 ${ }^{+}$Th2 response and associated immunoprotection in an adoptive-transfer murine model. J. Gen. Virol. 90, 818-826 (2009).
40. Weiskopf, D. et al. Comprehensive analysis of dengue virus-specific responses supports an HLA-linked protective role for $\mathrm{CD}^{+} \mathrm{T}$ cells. Proc. Natl Acad. Sci. USA 110, E2046-E2053 (2013).

This study shows that the breadth and magnitude of the anti-DENV $\mathrm{CD8}^{+} \mathrm{T}$ cell response in humans are associated with specific HLA alleles and a protective role for this cell population against DENV.

41. Hatch, S. et al. Intracellular cytokine production by dengue virus-specific $\mathrm{T}$ cells correlates with subclinical secondary infection. J. Infect. Dis. 203, 1282-1291 (2011).

42. Weiskopf, D. et al. Dengue virus infection elicits highly polarized CX3CR1 ${ }^{+}$ cytotoxic $\mathrm{CD}^{+}{ }^{+} \mathrm{T}$ cells associated with protective immunity. Proc. Natl. Acad. Sci. USA 112, E4256-E4263 (2015).

43. Kohler, S. et al. The early cellular signatures of protective immunity induced by live viral vaccination. Eur. J. Immunol. 42, 2363-2373 (2012).

44. Cimini, E. et al. Human Zika infection induces a reduction of IFN- $\gamma$ producing CD4 T-cells and a parallel expansion of effector V82 T-cells. Sci. Rep. 7, 6313 (2017).

45. Quaresma, J. A. et al. Hepatocyte lesions and cellular immune response in yellow fever infection. Trans. R. Soc. Trop. Med. Hyg. 101, 161-168 (2007).

46. Mongkolsapaya, J. et al. Original antigenic sin and apoptosis in the pathogenesis of dengue hemorrhagic fever. Nat. Med. 9, 921-927 (2003). This study shows original antigenic sin in dengue infection, in which many DENV-specific $T$ cell responses during secondary dengue had low affinity for the infecting serotype but higher affinity to other serotypes, probably the previously infecting serotype.

47. Gagnon, S. J., Ennis, F. A. \& Rothman, A. L. Bystander target cell lysis and cytokine production by dengue virus-specific human $\mathrm{CD} 4^{+}$cytotoxic T-lymphocyte clones. J. Virol. 73, 3623-3629 (1999).

48. Webster, R. G. Disquisitions of original antigenic sin. I. Evidence in man. J. Exp. Med. 124, 331-345 (1966).

49. Mangada, M. M. et al. Dengue-specific T cell responses in peripheral blood mononuclear cells obtained prior to secondary dengue virus infections in Thai schoolchildren. J. Infect. Dis. 185, 1697-1703 (2002).

50. Talarico, L. B. et al. The role of heterotypic DENV-specific CD8 ${ }^{+} \mathrm{T}$ lymphocytes in an immunocompetent mouse model of secondary dengue virus infection. EBioMedicine 20, 202-216 (2017).

This study shows that cross-reactive $T$ cell responses generated from the first infection contribute to the development of hemorrhagic disease in mice secondarily infected with the other serotype.

51. Elong Ngono, A. et al. Protective role of cross-reactive CD8 T cells against dengue virus infection. EBioMedicine 13, 284-293 (2016).

52. Zellweger, R. M. et al. CD8 ${ }^{+} \mathrm{T}$ cells can mediate short-term protection against heterotypic dengue virus reinfection in mice. J. Virol. 89, 6494-6505 (2015)

This paper addresses the contribution of cross-reactive anti-DENV $T$ cell responses and shows that this type of immunity is required to protect against infection with a heterotypic DENV serotype but not against homotypic reinfection.

53. Wen, J. et al. Identification of Zika virus epitopes reveals immunodominant and protective roles for dengue virus cross-reactive $\mathrm{CD}^{+} \mathrm{T}$ cells. Nat. Microbiol. 2, 17036 (2017). The results presented in this paper not only identify DENV and ZIKV cross-reactive epitopes but also show that the $\mathrm{T}$ cell response against ZIKV is altered in DENV-immune and naïve mice.

54. $\mathrm{Li}$, J. et al. Cross-protection induced by Japanese encephalitis vaccines against different genotypes of Dengue viruses in mice. Sci. Rep. 6, 19953 (2016).

55. Stettler, K. et al. Specificity, cross-reactivity, and function of antibodies elicited by Zika virus infection. Science 353, 823-826 (2016). This paper provides in vivo evidence of serological interaction between DENV and ZIKV, demonstrating that antibodies to ZIKV can enhance DENV infection in mice.

56. Zhao, H. et al. Structural basis of Zika virus-specific antibody protection. Cell 166, 1016-1027 (2016).

57. Beltramello, M. et al. The human immune response to Dengue virus is dominated by highly cross-reactive antibodies endowed with neutralizing and enhancing activity. Cell Host Microbe 8, 271-283 (2010).

Through analysis of samples from DENV-infected patients, this study shows that the human antibody response is dominated by cross-reactive antibodies to EDI/DII and prM.

58. Throsby, M. et al. Isolation and characterization of human monoclonal antibodies from individuals infected with West Nile Virus. J. Virol. 80, 6982-6992 (2006).

59. Wahala, W. M., Kraus, A. A., Haymore, L. B., Accavitti-Loper, M. A. \& de Silva, A. M. Dengue virus neutralization by human immune sera: role of envelope protein domain III-reactive antibody. Virology 392, 103-113 (2009).

60. Robbiani, D. F. et al. Recurrent potent human neutralizing antibodies to Zika virus in Brazil and Mexico. Cell 169, 597-609.e511 (2017). 
61. Yu, L. et al. Delineating antibody recognition against Zika virus during natural infection. JCI Insight 2, e93042 (2017).

62. Dejnirattisai, W. et al. Dengue virus sero-cross-reactivity drives antibodydependent enhancement of infection with zika virus. Nat. Immunol. 17, 1102-1108 (2016).

63. Nelson, S. et al. Maturation of West Nile virus modulates sensitivity to antibody-mediated neutralization. PLoS Pathog. 4, e1000060 (2008).

64. Fibriansah, G. \& Lok, S. M. The development of therapeutic antibodies against dengue virus. Antiviral Res. 128, 7-19 (2016).

65. Teoh, E. P. et al. The structural basis for serotype-specific neutralization of dengue virus by a human antibody. Sci. Transl. Med. 4, 139ra83 (2012).

66. de Alwis, R. et al. In-depth analysis of the antibody response of individuals exposed to primary dengue virus infection. PLoS Negl. Trop. Dis. 5, e1188 (2011).

67. Fibriansah, G. et al. A highly potent human antibody neutralizes dengue virus serotype 3 by binding across three surface proteins. Nat. Commun. 6, 6341 (2015).

68. Fibriansah, G. et al. A potent anti-dengue human antibody preferentially recognizes the conformation of $\mathrm{E}$ protein monomers assembled on the virus surface. EMBO Mol. Med. 6, 358-371 (2014).

69. Fibriansah, G. et al. Cryo-EM structure of an antibody that neutralizes dengue virus type 2 by locking E protein dimers. Science 349, 88-91 (2015).

70. Qiu, X. et al. Structural basis for neutralization of Japanese encephalitis virus by two potent therapeutic antibodies. Nat. Microbiol. 3, 287-294 (2018).

71. Hasan, S. S. et al. A human antibody against Zika virus crosslinks the $\mathrm{E}$ protein to prevent infection. Nat. Commun. 8, 14722 (2017)

72. Kaufmann, B. et al. Neutralization of West Nile virus by cross-linking of its surface proteins with Fab fragments of the human monoclonal antibody CR4354. Proc. Natl. Acad. Sci. USA 107, 18950-18955 (2010).

73. Rouvinski, A. et al. Recognition determinants of broadly neutralizing human antibodies against dengue viruses. Nature 520, 109-113 (2015).

74. Abbink, P. et al. Therapeutic and protective efficacy of a dengue antibody against Zika infection in rhesus monkeys. Nat. Med. 24, 721-723 (2018).

75. Barba-Spaeth, G. et al. Structural basis of potent Zika-dengue virus antibody cross-neutralization. Nature 536, 48-53 (2016).

76. Smith, S. A. et al. Persistence of circulating memory B cell clones with potential for dengue virus disease enhancement for decades following infection. J. Virol. 86, 2665-2675 (2012).

77. Junjhon, J. et al. Differential modulation of prM cleavage, extracellular particle distribution, and virus infectivity by conserved residues at nonfurin consensus positions of the dengue virus pr-M junction. J. Virol. 82, 10776-10791 (2008).

78. Watterson, D., Modhiran, N. \& Young, P. R. The many faces of the flavivirus NS1 protein offer a multitude of options for inhibitor design. Antiviral Res. 130, 7-18 (2016)

79. Modhiran, N. et al. Dengue virus NS1 protein activates cells via Toll-like receptor 4 and disrupts endothelial cell monolayer integrity. Sci. Transl. Med. 7, 304ra142 (2015).

80. Puerta-Guardo, H., Glasner, D. R. \& Harris, E. Dengue virus NS1 disrupts the endothelial glycocalyx, leading to hyperpermeability. PLoS Pathog. 12, e1005738 (2016).

81. Schlesinger, J. J., Brandriss, M. W. \& Walsh, E. E. Protection against 17D yellow fever encephalitis in mice by passive transfer of monoclonal antibodies to the nonstructural glycoprotein gp48 and by active immunization with gp48. J. Immunol. 135, 2805-2809 (1985).

82. Henchal, E. A., Henchal, L. S. \& Schlesinger, J. J. Synergistic interactions of anti-NS1 monoclonal antibodies protect passively immunized mice from lethal challenge with dengue 2 virus. J. Gen. Virol. 69 , 2101-2107 (1988).

83. Chung, K. M. et al. Antibodies against West Nile Virus nonstructural protein NS1 prevent lethal infection through Fc gamma receptor-dependent and -independent mechanisms. J. Virol. 80, 1340-1351 (2006).

84. Beatty, P. R. et al. Dengue virus NS1 triggers endothelial permeability and vascular leak that is prevented by NS1 vaccination. Sci. Transl. Med. 7 , 304ra141 (2015)

This paper, together with refs. ${ }^{79,80}$, shows that DENV NS1 is involved in the development of vascular leakage in mice.

85. Chuang, Y. C., Lin, J., Lin, Y. S., Wang, S. \& Yeh, T. M. Dengue virus nonstructural protein 1 -induced antibodies cross-react with human plasminogen and enhance its activation. J. Immunol. 196, 1218-1226 (2016)

86. Chuang, Y. C., Lin, Y. S., Liu, H. S. \& Yeh, T. M. Molecular mimicry between dengue virus and coagulation factors induces antibodies to inhibit thrombin activity and enhance fibrinolysis. J. Virol. 88, 13759-13768 (2014)

87. Halstead, S. B., Chow, J. S. \& Marchette, N. J. Immunological enhancement of dengue virus replication. Nat. New Biol. 243, 24-26 (1973).

88. Halstead, S. B. \& O'Rourke, E. J. Antibody-enhanced dengue virus infection in primate leukocytes. Nature 265, 739-741 (1977).
89. Pierson, T. C. et al. The stoichiometry of antibody-mediated neutralization and enhancement of West Nile virus infection. Cell Host Microbe 1, 135-145 (2007).

90. Kliks, S. C., Nimmanitya, S., Nisalak, A. \& Burke, D. S. Evidence that maternal dengue antibodies are important in the development of dengue hemorrhagic fever in infants. Am. J. Trop. Med. Hyg. 38, 411-419 (1988).

91. Katzelnick, L. C. et al. Antibody-dependent enhancement of severe dengue disease in humans. Science 358, 929-932 (2017).

92. Hadinegoro, S. R. et al. Efficacy and long-term safety of a dengue vaccine in regions of endemic disease. N. Engl. J. Med. 373, 1195-1206 (2015).

93. Halstead, S. B. \& Russell, P. K. Protective and immunological behavior of chimeric yellow fever dengue vaccine. Vaccine 34, 1643-1647 (2016).

94. SAGE Working Group on Dengue Vaccines and WHO Secretariat. Background paper on dengue vaccines: revision to the background paper from 17 March 2016. SAGE Meeting of April 2018 http://www.who.int/ immunization/sage/meetings/2018/april/2_DengueBackgrPaper_SAGE_ Apr2018.pdf?ua=1 (2018)

95. de Alwis, R. et al. Dengue viruses are enhanced by distinct populations of serotype cross-reactive antibodies in human immune sera. PLoS Pathog. 10, e1004386 (2014)

96. Haslwanter, D., Blaas, D., Heinz, F. X. \& Stiasny, K. A novel mechanism of antibody-mediated enhancement of flavivirus infection. PLoS Pathog. 13, e1006643 (2017)

This work describes a new modality of ADE in which enhancement of viral entry is derived from an antibody-mediated increase in the exposure of the fusion loop and is independent of other cell-surface proteins.

97. Zellweger, R. M., Eddy, W. E., Tang, W. W., Miller, R. \& Shresta, S. CD8 ${ }^{+}$ $\mathrm{T}$ cells prevent antigen-induced antibody-dependent enhancement of dengue disease in mice. J. Immunol. 193, 4117-4124 (2014).

98. Fagbami, A. H., Halstead, S. B., Marchette, N. J. \& Larsen, K. Crossinfection enhancement among African flaviviruses by immune mouse ascitic fluids. Cytobios 49, 49-55 (1987).

99. Halstead, S. B., Porterfield, J. S. \& O’Rourke, E. J. Enhancement of dengue virus infection in monocytes by flavivirus antisera. Am. J. Trop. Med. Hyg. 29, 638-642 (1980).

100. Priyamvada, L. et al. Human antibody responses after dengue virus infection are highly cross-reactive to Zika virus. Proc. Natl. Acad. Sci. USA 113, 7852-7857 (2016).

101. Bardina, S. V. et al. Enhancement of Zika virus pathogenesis by preexisting antiflavivirus immunity. Science 356, 175-180 (2017).

102. George, J. et al. Prior exposure to Zika virus significantly enhances peak Dengue-2 viremia in rhesus macaques. Sci. Rep. 7, 10498 (2017).

103. McCracken, M. K. et al. Impact of prior flavivirus immunity on Zika virus infection in rhesus macaques. PLoS Pathog. 13, e1006487 (2017).

104. Halai, U. A. et al. Maternal Zika virus disease severity, virus load, prior dengue antibodies, and their relationship to birth outcomes. Clin. Infect. Dis. 65, 877-883 (2017).

105. Anderson, K. B. et al. Preexisting Japanese encephalitis virus neutralizing antibodies and increased symptomatic dengue illness in a school-based cohort in Thailand. PLoS Negl. Trop. Dis. 5, e1311 (2011).

106. Saito, Y. et al. Japanese encephalitis vaccine-facilitated dengue virus infection-enhancement antibody in adults. BMC Infect. Dis. 16, 578 (2016).

107. Chan, K. R. et al. Cross-reactive antibodies enhance live attenuated virus infection for increased immunogenicity. Nat. Microbiol. 1, 16164 (2016).

108. Scherwitzl, I., Mongkolsapaja, J. \& Screaton, G. Recent advances in human flavivirus vaccines. Curr. Opin. Virol. 23, 95-101 (2017).

109. Kirkpatrick, B. D. et al. The live attenuated dengue vaccine TV003 elicits complete protection against dengue in a human challenge model. Sci. Transl. Med. 8, 330ra36 (2016).

110. Guy, B. et al. From research to phase III: preclinical, industrial and clinical development of the Sanofi Pasteur tetravalent dengue vaccine. Vaccine 29, 7229-7241 (2011).

111. Sabchareon, A. et al. Protective efficacy of the recombinant, live-attenuated, CYD tetravalent dengue vaccine in Thai schoolchildren: a randomised, controlled phase 2b trial. Lancet 380, 1559-1567 (2012).

112. Capeding, M. R. et al. Clinical efficacy and safety of a novel tetravalent dengue vaccine in healthy children in Asia: a phase 3, randomised, observer-masked, placebo-controlled trial. Lancet 384, 1358-1365 (2014).

113. Villar, L. et al. Efficacy of a tetravalent dengue vaccine in children in Latin America. N. Engl. J. Med. 372, 113-123 (2015).

114. Sridhar, S. et al. Effect of dengue serostatus on dengue vaccine safety and efficacy. N. Engl. J. Med. 379, 327-340 (2018).

This study on cumulative safety and efficacy confirms the higher risk of severe dengue among seronegative Dengvaxia recipients than among seronegative vaccine controls.

115. Lima, N. S., Rolland, M., Modjarrad, K. \& Trautmann, L. T cell immunity and Zika virus vaccine development. Trends Immunol. 38, 594-605 (2017). 
116. Guy, B. \& Jackson, N. Dengue vaccine: hypotheses to understand CYD-TDV-induced protection. Nat. Rev. Microbiol. 14, 45-54 (2016).

117. Ishikawa, T., Yamanaka, A. \& Konishi, E. A review of successful flavivirus vaccines and the problems with those flaviviruses for which vaccines are not yet available. Vaccine 32, 1326-1337 (2014).

118. Brault, A. C. et al. A Zika vaccine targeting NS1 protein protects immunocompetent adult mice in a lethal challenge model. Sci. Rep. 7, 14769 (2017)

119. Ishikawa, T. et al. Enhancing the utility of a prM/E-expressing chimeric vaccine for Japanese encephalitisby addition of the JEV NS1 gene.Vaccine 29, 7444-7455 (2011).

120. Chen, M. C. et al. Deletion of the C-terminal region of dengue virus nonstructural protein 1 (NS1) abolishes anti-NS1-mediated platelet dysfunction and bleeding tendency. J. Immunol. 183, 1797-1803 (2009).

121. Zheng, A., Umashankar, M. \& Kielian, M. In vitro and in vivo studies identify important features of dengue virus pr-E protein interactions. PLoS Pathog. 6, e1001157 (2010).

122. Crill, W. D. et al. Sculpting humoral immunity through dengue vaccination to enhance protective immunity. Front. Immunol. 3, 334 (2012).

123. Richner, J. M. et al. Modified mRNA vaccines protect against Zika virus infection. Cell 168, 1114-1125.e1110 (2017).
124. Rouvinski, A. et al. Covalently linked dengue virus envelope glycoprotein dimers reduce exposure of the immunodominant fusion loop epitope. Nat. Commun. 8, 15411 (2017).

125. Slon Campos, J. L. et al. Temperature-dependent folding allows stable dimerization of secretory and virus-associated E proteins of Dengue and Zika viruses in mammalian cells. Sci. Rep. 7, 966 (2017).

\section{Acknowledgements}

This work was supported by the Wellcome Trust, UK, and the Newton-Medical Research Council, UK. G.R.S. is supported as a Wellcome Trust Senior Investigator.

\section{Competing interests}

The authors declare no competing interests.

\section{Additional information}

Reprints and permissions information is available at www.nature.com/reprints.

Correspondence should be addressed to G.R.S.

Publisher's note: Springer Nature remains neutral with regard to jurisdictional claims in published maps and institutional affiliations. 